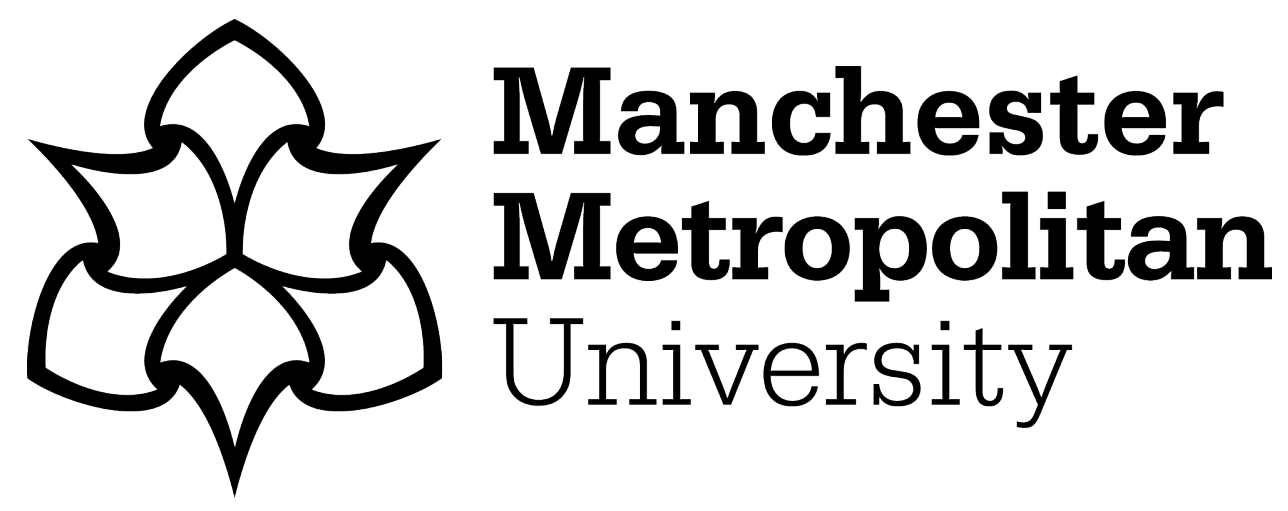

Williams, L, Ralphs, R ORCID logoORCID: https://orcid.org/0000-00018359-2598 and Gray, PM (2017) The Normalisation of Cannabis Use Among Bangladeshi and Pakistani Youth: A New Frontier for the Normalisation Thesis? Substance Use and Misuse, 52 (4). pp. 413-421. ISSN 1082-6084

Downloaded from: https://e-space.mmu.ac.uk/617309/

Version: Accepted Version

Publisher: Taylor \& Francis

DOI: https://doi.org/10.1080/10826084.2016.1233565

Please cite the published version 


\title{
The Normalisation of Cannabis Use Among Bangladeshi and Pakistani Youth: A New Frontier for the Normalisation Thesis?
}

\author{
Lisa Williams, Rob Ralphs and Paul Gray
}

\begin{abstract}
Background: The Asian population in Britain has grown, representing the second largest ethnic group; Bangladeshi, Pakistani and Indian nationalities are prevalent (Jivraj, 2012; ONS, 2012). Yet, we know relatively little about the nature and extent of their substance use. Jayadowsky (2006) argues ethnic minority groups may be influenced by the norms and values of the dominant culture. Given recreational drug use has undergone a process of normalisation in Britain (Aldridge et al., 2011; Parker et al, 1998, 2002), we explore the degree to which this is occurring in a Bangladeshi and Pakistani community of Muslim faith in Northern England; a group typically assumed to reject substance use because of robust religious and cultural values. Objectives: To examine the extent, frequency and nature of substance use, and associated attitudes. Methods: A cross-sectional study collecting qualitative data from a sample $(\mathrm{N}=43)$ of adolescents accessing a drug service and a range of professionals working with them during 2014. We also present analyses of routinely collected quantitative client data. Results: Adolescent interviewees reported extensive personal experience smoking skunk cannabis, and professionals working in the community confirmed many young Asians smoked it. Its consumption appeared to be accommodated into the daily lives of young people and the supply of it also showed signs of acceptance. Conclusions: Skunk cannabis may be undergoing a process of normalisation within some Asian communities in Britain. Our study has significant implications for the normalisation thesis, finding evidence for normalisation within a subpopulation that is typically perceived to resist this trend.
\end{abstract}

\section{INTRODUCTION}

The composition of the population in England and Wales has become more ethnically diverse with Asian/Asian British residents now being the second largest group after the White/White British population (ONS, 2012). The most common Asian nationalities are Indian, Pakistani and Bangladeshi with recent increases being greatest in Pakistani and Bangladeshi communities (Jivraj, 2012; ONS, 2012). These groups have high proportions of young people compared to the White British population (Centre for Policy and Ageing, 2013; ONS, 2013), and drug use is associated with young people (Aldridge et al., 2011). They are also more likely to be of Muslim faith (The Muslim Council of Britain, 2015), a religion that proscribes the consumption of intoxicants (Safian, 2013). Furthermore, they tend to live in deprived neighbourhoods afflicted by poor housing, inadequate education, limited access to health services, low income and unemployment (Jivraj and Khan, 2013), many factors also linked with some types of drug use (see Pearson and Patel, 1998). Given these population changes, the sizeable youth population among Asian groups, and assertions that recreational drug use has undergone a process of normalisation in Britain (see Aldridge et al., 2011), it is somewhat surprising that British drugs researchers have overlooked these communities, especially since Jayadowsky (2006) argued ethnic minority groups may be influenced by the norms and values of the wider British culture.

There are various reasons for this oversight. In understanding and explaining recreational substance use, research justifiably tends to focus on specific social groups with relatively high levels of consumption, often using samples with predominantly White participants. Some can 
be described as spectacular drugs consumers, for example, those who frequent nightclubs (see Measham et al., 2001; Moore et al., 2013), others include those in drug treatment (see Best et al., 2009; Seddon et al., 2012), all atypical of the majority of drug users. ${ }^{i}$ Moreover, in comparison to all other ethnic groups, national self-report surveys find Asian British communities in England and Wales have the lowest levels of drug use (see HSCIC, 2015; Lader, 2015). Even though these data are likely to under-estimate prevalence (Fountain et al., 2003; UKDPC, 2010; Wanigaratne et al., 2003), they may partly explain why the Asian British community have been neglected by British drugs researchers. Furthermore, despite Asian British respondents featuring in national surveys as part of a representative sample, there is a lack of small scale British research focusing solely on these communities and their illegal drugs consumption. Since many British Asians are Muslim and their religion proscribes the use of intoxicants, ${ }^{\text {ii }}$ the shame and stigma attached to substance use (see Brady, 2007; Fountain et al., 2003) may mean they are unlikely to engage with drugs researchers and/or admit to it, further complicating this problem (see Pearson and Patel, 1998). This led Patel and Wibberley (2002:51) to bemoan the 'paucity of knowledge' in respect of substance use, particularly patterns of consumption, among ethnic minority groups (see also Fountain et al., 2003; UKDPC, 2010), and little has changed since.

Although current research is sparse, we outline what we can glean about alcohol and illegal drug use within young, predominantly adolescent, South Asians of Bangladeshi, Indian or Pakistani origin living in Britain. Compared to other ethnic groups, the prevalence of alcohol consumption is low among these communities (see Best et al., 2001; Best et al., 2005; HSCIC, 2015; Jawad et al., 2014). Gender differences exist with males more likely to be regular drinkers (Rodham et al., 2005). In 2014, the Smoking, Drinking and Drug Use Surveys collecting data from a representative sample of English adolescents aged 11-15 (see HSCIC, 2015), estimated 13 per cent of Asian adolescents had tried drugs at least once. This was similar to other ethnic groups, particularly White respondents, which stood at 14 per cent. Compared to other ethnic groups, regular consumption was lowest among Asian adolescents. A small minority were regular users: six per cent in the past year; four per cent in the past month. Similar to alcohol consumption, males were more likely to be drug users (see also Rodham et al., 2005). The Crime Survey for England and Wales (CSEW - Home Office, 2014) has also found lower rates of drug taking among Asian ethnicities in comparison to other ethnic groups. Further, it demonstrated how regular consumption is associated with young British Asians, whereas for White ethnicities it is more evenly distributed across the age range. The highest rates of regular drugs consumption, compared to other Asian ethnicities, were reported by Bangladeshi and Pakistani respondents aged 16-24. Their patterns of drug use were similar to the general population with cannabis being most common (see also Fountain, 2009; Fountain et al., 2003) and drugs like ecstasy, cocaine and heroin comprising drug-taking repertoires for a minority (see also Jayakody et al., 2006). Patterns of use among Asian communities may also be distinct. Jayakody et al. (2006) found the highest prevalence of paan $^{\mathrm{iii}}$ and solvent/gas consumption was among Asian British adolescents compared to White and other ethnic groups. Paan use was predominantly associated with those of Bangladeshi origin. The data summarised demonstrates that in respect of illegal drug use, young British Asians may not be too different from their White British counterparts. As Seddon (2006) argued, some ethnic minority groups are 'catching up' with their White counterparts in relation to their substance use. Even though regular consumption may be lower compared to other ethnic groups, the data summarised demonstrates a willingness for these communities to experiment with illegal drugs. Coupled with the findings we present here, they lead us to contemplate whether recreational drug use is undergoing a process of normalisation (see Aldridge et al., 2011; Parker et al., 1998, 2002) within some British (Muslim) Asian communities. 
The concept of the normalisation of drug use first emerged in Britain during the 1990s when the availability of a range of substances expanded, matched by a sharp increase in recreational drug use, and a wider acceptance of it. The normalisation thesis (Aldridge et al., 2011; Parker et al., 1998, 2002), originating from the Illegal Leisure longitudinal study, beginning in 1991, offered an explanation for the increasing drug consumption trends recorded among its sample and also documented by British government surveys from the mid-1990s to the millennium (see Williams, 2016). This enduring thesis identified how recreational drug use was undergoing a process of normalisation whereby it was being accommodated into the lives of many young people, whether or not they were drug users. Evidence for normalisation (see Parker et al., 1998; 2002) included the widespread availability and relatively easy accessibility of recreational drugs; a growth in the numbers of young people willing to try them, as well as an increase in regular users, and connected to this, that young people from a range of different backgrounds were equally likely to become drug users, ${ }^{\text {iv }}$ tolerance of recreational drug users by some non-drug users; and increasing cultural accommodation leading to recreational drugs and drug users infusing many aspects of daily life and being subject to less condemnation than previously. Normalisation, then, is concerned with the 'accommodation of psychoactive experiences into everyday life' (Aldridge et al., 2011: 226), whether it be through personal recreational drugs consumption, being drugs aware, knowing recreational drug users and being tolerant of them, or because of an increase in positive mass media representations of drugs and drug users.

Although the normalisation thesis is not without its critics (see Shiner and Newburn, 1997; 1999), research has found evidence for normalisation among young offenders (Hammersley et al., 2003), the dance music scene (Measham et al., 2001), in other cultures (Duff, 2005; RodnerSznitman, 2008), older populations (Pearson, 2001) and of the social supply of drugs (Coomber et al., 2015). Shildrick (2002) refined the concept addressing its perceived homogeneity, in particular, how it appeared to fail to distinguish between the different experiences and attitudes of young people. She coined the term 'differentiated normalisation', asserting recreational drug use is not normalised for some young people. ${ }^{\mathrm{V}}$ Given their strong religious and cultural beliefs, Muslim Bangladeshi and Pakistani communities represent one such group for which recreational drug use may be perceived not to be normalised. In Britain, there has been a lack of research applying the normalisation concept to different ethnic groups. ${ }^{\text {vi }}$ Our study aims to address this deficit exploring whether or not certain kinds of drug use indicate a process of normalisation within the Asian British population, particularly Muslim Bangladeshi and Pakistani communities. Typically, these groups may be seen to buck the normalisation trend. Our study also begins to address the deficit of current British drugs research with Asian communities.

\section{METHODS}

Our study evaluated an outreach programme for young people aged up to 21 in Mill Town, Northern England, located at Making A Difference, ${ }^{\text {vii }}$ a young person's alcohol and drug service. Mill Town comprised of three small communities and the Asian/Asian British population predominantly of Pakistani or Bangladeshi origin with high numbers of young people - was much greater than the national average, ranging between 75 and 82 per cent (ONS, 2013). Given the composition of the local community, Making A Difference focused upon engaging the young Asian population and the findings presented resulted from an evaluation of their outreach programme. The outreach team walked the streets two evenings a week and rather than spreading themselves too thinly, they targeted Pakistani and Bangladeshi communities in small geographical areas. Street-based outreach was complimented with 'in-reach' in schools, 
a pupil referral unit and colleges. Having a presence in educational settings was instrumental in engaging young females from these communities, who partner agencies acknowledged were less visible on the streets.

The research design employed a mixed methods approach collecting data between January and June 2014. ${ }^{\text {viii }}$ Semi-structured interviews were undertaken with staff working for Making $A$ Difference $(n=6)$, clients and one family member currently engaged with the programme $(n=19$; male clients, $n=16$; Muslim clients, $n=19)$, and representatives from external agencies $(n=18)$ including local education establishments, social and health services, and criminal justice organisations. The interview sample were recruited through case workers and research team members asking for volunteers as they met appropriate participants during the fieldwork period. The purpose of interviews with clients was to obtain a profile of their alcohol and drugs consumption, and their views about Making A Difference. A variety of Making A Difference staff were selected for interview from managers, outreach workers, advocacy and family workers, as well as a range of professionals working in partnership with them. These interviews focused on the perceived substance use issues for young Asians in the area, the effectiveness of the outreach service, and professional partnership relations. All interviews were analysed thematically, identifying the extent and nature of substance use, and perceived attitudes towards it and the availability of illegal drugs in Mill Town. We also analysed quantitative client data routinely collected by the project between November 2011 and October 2013. During this period, the project worked with 837 clients. Almost a tenth $(n=80)$ were Asian (Bangladeshi $n=21,26$ per cent; Pakistani $n=47,59$ per cent; and Asian Other $n=12,15$ per cent). They were predominantly male ( $n=68,85$ per cent) and the majority ( $n=64,80$ per cent) were aged 14 to 16 . In contrast, White referrals were more likely to be older. We present the qualitative interview data and some of the quantitative data in the following section.

\section{RESULTS}

\section{Bangladeshi and Pakistani adolescent substance use in Mill Town}

When clients accessed Making A Difference, they indicated whether they had a 'problem' solely with drugs, solely with alcohol or with both drugs and alcohol (see Table 1). ${ }^{\text {ix }}$ Compared to White clients, Asian clients rarely reported a 'problem' solely with alcohol, instead the vast majority indicated they had a 'problem' solely with drugs.

Table 1: Proportion of Asian/British Asian clients reporting a 'problem' with alcohol, drugs, or drugs and alcohol from November 2011 to October 2013

\begin{tabular}{|l|c|c|c|}
\hline 'Problem' with: & Females $(\mathrm{n}=12) \%$ & Males $(\mathrm{n}=68) \%$ & Total $(\mathrm{n}=80) \%$ \\
\hline Alcohol & 8.3 & 1.5 & 2.5 \\
\hline Drugs & 91.7 & 86.8 & 87.5 \\
\hline Drugs and Alcohol & 0.0 & 11.8 & 10.0 \\
\hline
\end{tabular}

Despite this, some professionals working with young Asians in Mill Town claimed alcohol consumption was common. They suggested, because of its 'forbidden' nature (see Brady, 2007), and the shame and stigma consumption might bring upon a family (see Fountain et al., 2003), it was likely to be concealed and, therefore, young Asians were not presenting at support services with it as a substance use issue:

... we have quite a high [level of use] locally, in the Pakistani community, and particularly around alcohol. That might be difficult for a young person to disclose, that 
they're having a problem with alcohol, and then to move it on into a service. They would be very anxious, probably, around confidentiality. (College Student Support Staff, ID 10)

In contrast, whilst some of our adolescent Asian interviewees admitted to trying alcohol, none divulged current use, and many discussed how alcohol was not a significant problem for their peers:

... one or two people may be seen on the streets drunk in the area, but drink isn't really a problem. (16 year old, Pakistani, male, ID SU15)

Examining prevalence of alcohol consumption offers support for the view that substance use might be low among Asian communities. However, when we turn our attention to illegal drug use, in particular, cannabis, a different picture emerges, one that suggests, unlike for alcohol, consumption may be widespread and accommodated among young Asians in Mill Town. Our findings in respect of cannabis offer support for the normalisation thesis within a subpopulation who historically have low levels of recreational drug use. The features of normalisation that were apparent to us corresponded with many of those identified by Parker et al. $(1998,2002)$ and Aldridge et al. (2011), including the perceived widespread availability and easy accessibility of cannabis, regular consumption of it among our sample and their friends or family, and a general acceptance of it by the wider community.

\section{The extent and frequency of illegal drug use: skunk cannabis}

In describing their illegal drug use to us, all adolescent interviewees discussed how they had tried cannabis and did not report trying any other illegal drugs or novel psychoactive substances. Given their age, cannabis consumption is to be expected (see Parker et al., 1998). Some did note how cocaine and heroin use occurred in their community, however, it was associated with older adolescents and adults. When they talked about their consumption of cannabis, many discussed how it was a particular strain of cannabis that was widespread in Mill Town, skunk. ${ }^{\mathrm{x}}$ The different types mentioned by interviewees were Lemon Haze, Dynamite Haze and Blue Cheese. The professionals we interviewed also confirmed skunk cannabis was dominant:

Young people are predominantly using skunk and for them, this is just standard cannabis. It's all they know. (Making A Difference Staff, ID 3)

The normalisation thesis argued cannabis offered the strongest support for normalisation (Parker et al., 2002, see also Shildrick, 2002). However, Parker et al. (2002) also asserted skunk cannabis, because of its possible negative side effects and adverse media attention, was less likely to be acceptable. Now, skunk use among British youth is commonplace (see Lader, 2015), a development evidenced by our sample.

Normalisation describes the degree to which recreational drug use is perceived to be acceptable in society. The extent of drug trying and regular use are important indicators (Aldridge et al., 2011; Parker et al., 1998, 2002). Although normalisation is not concerned with absolutes - that is, a certain number of the population must be drug users for normalisation to occur (Aldridge et al., 2011; Parker et al., 1998, 2002) - studies supporting drug normalisation in particular settings have found large proportions of drug users (see Hammersley et al., 2003; Measham et al., 2001). As we have already shown, all our adolescent interviewees consumed skunk 
cannabis. Many also confirmed the majority of their friends smoked it, a perception supported by the professionals we interviewed:

I'd say about seven, eight out of ten [of my friends] smoke weed [cannabis]. (16 year old, Pakistani, male, ID SU4)

More or less all of them [smoke cannabis] ... (15 year old, Pakistani, male, ID SU11)

... these young people haven't got any friends that don't use [skunk cannabis] ... (Making A Difference Staff, ID 2)

A professional working in a school with predominantly Asian students noted:

Imean, we've got, I think, 950 kids in our school and if somebody came to me and said, "How many of them smoke cannabis?" I would probably say, "More than half." (School Student Support Staff, ID 13)

It appears, then, that cannabis consumption was widespread within the young Asian community in Mill Town. When our adolescent interviewees described their history of consumption, all discussed how they had been daily cannabis smokers, and many still were. The quantities consumed varied, with some noting they smoked one joint per day and others as many as ten. The widespread consumption of cannabis and its regular use among our adolescent interviewees, together with perceptions that its consumption was prevalent among young people in the wider community in Mill Town, all point towards the normalisation of this substance.

\section{The profile of skunk cannabis users and the context of use}

The age of onset of skunk cannabis use indicates how it has become acceptable for young teenagers to consume it in Mill Town. The majority of the professionals working in Mill Town believed the onset of cannabis use had changed and was beginning at an earlier age in the Asian community:

They're getting younger, that's the issue.... It used to be probably Year 10 or 11s [aged 14-16], mainly. Now its Year 8s [aged 12-13], 8s in particular, at the moment. (School Student Support Staff, ID 13)

Some of our adolescent interviewees confirmed they started smoking skunk cannabis as young as age 11. Our analysis of the quantitative data provided by Making A Difference revealed Asian clients were younger than their White counterparts and predominantly aged between 14 and 16, suggesting a younger age of onset of substance use or their consumption escalated quicker, requiring advice and support. Given the early onset of cannabis consumption for some of our interviewees, and the frequency of their consumption, this meant some had been smoking it daily for at least a year or two, and even longer.

The normalisation thesis emphasised how key demographics, such as gender, class and ethnicity no longer determined involvement in recreational drug use (see Parker et al., 1998). For example, young females and males were equally likely to become drug users. However, some of the authors of the original thesis have since claimed these demographics may still influence decisions about drugs (see Aldridge et al., 2011). In our research, more males than 
females were accessing Making A Difference, suggesting that cannabis consumption may be more prevalent among the young Asian male community in Mill Town and, therefore, that gender is an important determinant of drug consumption in this community (see Table 1). However, for those attending Making A Difference, gender differences were small; males and females were equally likely to report a 'problem' with drugs. Our adolescent interviewees included two female friends who previously smoked cannabis daily on the way to school and, at the time of interview, were smoking it occasionally, usually at weekends. Others discussed how cannabis, as well as alcohol consumption was not unusual among young Asian females:

You do get females as well, not as many, but yes, you certainly get Asian females drinking and smoking drugs. (Youth and Community Worker, ID 8)

... a lot of females are smoking [cannabis] around here, yeah, a lot. And they go into town and drink and that. (15 year old, Pakistani, male, ID SU11)

These findings suggest that the relationship between gender and skunk cannabis consumption in the Asian community requires additional investigation to establish the degree to which it is differentiated by gender.

Further evidence for normalisation was provided when our interviewees discussed when and where cannabis was smoked. As the normalisation thesis has shown, for many, recreational drug use is accommodated into daily lives and, for some, recreational drugs are often taken on special occasions (see Aldridge et al., 2011; Parker et al., 1998, 2002). Our data also supported these ideas. Usually, when our adolescent interviewees smoked cannabis, they told us they did so with their friends on the way to or from school, or after school. Staff we interviewed in schools also confirmed this was common. Furthermore, some discussed how they smoked it on special occasions, to celebrate, and in some cases, their consumption increased:

... if it's a special occasion, like, last Eid, I smoked about forty spliffs.

Forty spliffs? Over the Eid period?

Yeah. We are in our pad, innit, sitting there. (16 year old, Bangladeshi, male, ID SU5)

These findings illustrate how skunk cannabis consumption is accommodated within the lives of our adolescent interviewees suggesting it is becoming normalised among the young Asian community in Mill Town. To further assess the extent of normalisation, we turn to consider access and availability.

\section{Access to and availability of skunk cannabis}

The widespread availability and easy accessibility of recreational drugs during the 1990s and into the millennium in Britain provided further evidence towards normalisation (Aldridge et al., 2011; Parker et al., 1998, 2002). In Mill Town, cannabis was perceived to be easily accessible by our adolescent interviewees:

It's [cannabis] everywhere, it's increasing. ... It's easy to gain access ... because like everyone's smoking [cannabis], even the people younger than me as well, they're smoking it. People older than me, they just supply it, just like that. ... You just walk around the block and see someone there and just go ask him, he'll give it to you. (15 year old, Bangladeshi, male, ID SU2) 
Skunk is quicker to get hold of than a pizza delivery! (15 year old, Bangladeshi, male, ID SU8)

This idea that skunk cannabis was easy to obtain was supported by professionals working in the community. Some believed its production was firmly established in Mill Town, especially among the Asian community:

The thing is, with these little mill towns, there's loads of people growing it, you know, there's loads and loads of people renting factories, renting houses. When we were on outreach you could smell it. (Making A Difference Staff, ID 3)

In my experience a lot of cultivation of drugs or selling of drugs is [sic] the majority are Asian, and I never experienced it to the extent that it is before. (Criminal Justice Staff, ID 22)

It was apparent, then, that skunk cannabis was easily accessible for our adolescent interviewees and their peers, providing further support towards normalisation. Interviewees also suggested that access to it had increased recently. This was likely due to it being locally produced, rather than imported, and, as we shall see in the next section, facilitated by tolerance of local supply networks.

\section{Attitudes towards the supply and consumption of skunk cannabis in Mill Town}

Further criteria for the normalisation of recreational drug use include the extent to which attitudes towards drug use appear to be tolerant (Parker et al., 1998, 2002). Although Islam proscribes the consumption of any intoxicants (see Safian, 2013), skunk cannabis did not appear to be subject to disapproval in Mill Town, at least among younger members of the community:

Skunk is not haram. ${ }^{x i}$ (15 year old, Pakistani, Male, ID SU11)

Weed's [cannabis] like a normal thing for me now. (15 year old, Bangladeshi, male, ID SU8).

Like cannabis is not seen as a drug any more, it's just as, "Oh, I'm having a spliff - big deal." (Youth and Community Worker, ID 18)

Coomber et al (2015) have found evidence for the normalisation of social supply of recreational drugs. Similarly, in Mill Town, supplying skunk cannabis was perceived to be tolerated:

... there's a lot more, especially in the Asian community, young people selling drugs at school than there's ever been. ... Before in that community, if anyone was doing something like that, the whole community would know about it and they'd be stigmatised ... (Making A Difference Staff, ID 3)

However, different attitudes towards drugs within families could still cause potential conflict. About half of our young Asian interviewees confirmed they had not told their family they were accessing services at Making A Difference, suggesting their family might disapprove of their drug use. Nevertheless, there was also evidence that some families did not condemn cannabis use and different family members smoked it: 
... there's almost an acceptance now that so many families we work with, there's quite a high level of cannabis use from all family members. (College Student Support Staff, ID 10)

All my cousins smoke weed [cannabis]. (15 year old, Pakistani, female, ID SU10)

These different perceptions provide evidence towards differentiated normalisation (see Shildrick, 2002), suggesting that in some families and parts of the community, cannabis appeared to be accepted. Accommodating attitudes towards cannabis facilitated its consumption. As many of our adolescent interviewees emphasised earlier, most of their friends smoked it, which impacted upon their own decisions to smoke it (see also Williams, 2013). However, as Parker et al. (2002) found, even those who did not smoke skunk cannabis, tolerated its consumption by their friends:

... [a] couple of them [friends] smoke weed [cannabis] but some of them just stand there and do their own stuff, not smoking.

They don't kind of get under pressure to smoke?

No. Well they have been [cannabis] smokers before, but they have stopped it. (16 year old, Pakistani, male, ID SU15)

For Parker et al (2002) the acceptance of cannabis by drug abstainers and ex-users was an important indicator of normalisation. As the interviewees have highlighted here, at best, the perceived different attitudes towards the consumption of cannabis suggest at least a process of differentiated normalisation may be occurring in Mill Town.

\section{DISCUSSION}

Our findings signify an important breakthrough in respect of the normalisation debate. Support for the original thesis was offered by research with, for example, young offenders and clubbers (see Hammersley et al., 2003; Measham et al., 2001) - what we describe as the 'usual suspects', in particular settings or social groups. Shildrick's (2002) refinement of the concept highlighted how recreational drug use might be normalised for some groups of young people and not others. Our study has found evidence pointing towards normalisation in a subpopulation traditionally associated with robust anti-substance use attitudes grounded in religion and culture, a group who may be perceived to resist the normalisation trend. It is not surprising to us to find extremely experienced cannabis consumers among a sample accessing a drugs service, however, our findings demonstrate that professionals working in the wider community also perceived the consumption of skunk cannabis to be widespread and culturally accommodated in Mill Town.

It has been argued that patterns of substance use in Asian communities are similar to that of the White population (Fountain, 2009; Fountain et al., 2003). Our research supports this contention, showing, in line with the CSEW (see Lader, 2015), how the consumption of skunk cannabis is common among young people, in this case, of Bangladeshi and Pakistani origin, and Muslim faith. Our sample's drug use was distinctive, primarily involving one substance, skunk cannabis. Its widespread consumption challenges the conclusion, reached by Parker et al. in 2002, about it being unacceptable. Behaviour and attitudes appear to have changed. As well as extensive personal experience with skunk cannabis among our adolescent interviewees, there was evidence pointing towards wider acceptance of it in Mill Town. Many adolescent interviewees discussed how their friends or family smoked it, and professionals working in the 
community confirmed many young Asians did so, but were not accessing Making A Difference. There was also evidence suggesting its consumption was being accommodated within young Asian's daily lives and it was transcending gender boundaries. Furthermore, skunk cannabis was perceived to be easily accessible, with supply showing signs of acceptance, rather than condemnation. Although attitudes could differ among generations, with some family members censuring consumption, it seems young Bangladeshis and Pakistanis in Mill Town may tolerate its consumption, whether or not they smoke it themselves. These different attitudes may be a result of a shared, generic youth culture and young Asians becoming more westernised (see Patel and Wibberley, 2002).

In Mill Town, the age of onset of skunk cannabis use was perceived to be lowering: many of our interviewees had smoked it daily for a couple of years and some were smoking large quantities. Interviewees also discussed how cannabis was consumed on the way to school, perhaps indicating a shift in consumption patterns connected to recreation and leisure, to one that has the potential to interfere with studies. These are pressing policy concerns, especially as research has found regular and 'heavy' consumption of cannabis at a young age can have adverse effects upon mental health and development (see Casadio et al., 2011; Rubino and Parolaro, 2008; Shapiro and Buckley-Hunter, 2010).

Our research demonstrates the potential for the normalisation of skunk cannabis within other Asian communities in Britain. Moreover, with the mass migration of different Muslim groups from Arab states and Africa across Europe and into Britain, we may witness this trend within these populations in the future. Indeed, Mauseth et al. (2015) have found young Muslims in Jordan are beginning to accommodate cannabis consumption. Further research with Asian Muslim communities is required to estimate prevalence and answer questions relating to onset, persistence, desistance, drug preferences, and the role alcohol plays in young people's lives. It was somewhat surprising to us the extent to which skunk cannabis, compared to the consumption of alcohol, appeared acceptable to young Asians living in Mill Town. Further investigation of different attitudes towards different substances, and how they facilitate or constrain consumption, is necessary. Future research should also focus on communities where there is a concentration of Asians. As we noted previously, the Asian population in Britain has a greater proportion of young people, and in areas where these communities are clustered, as they were in Mill Town, we may find larger numbers of young people consuming substances. While Bangladeshis and Pakistanis were over-represented in Mill Town, they were under-represented at Making A Difference. This is consistent with the national picture: young Bangladeshis and Pakistanis are under-represented in the treatment population, currently comprising 2.5 per cent (NDTMS, 2015). In future, these communities may find they are faced with a range of substance use issues to which drug services need to respond. Our research focused on the Bangladeshi and Pakistani community, however, the British and Asian communities worldwide are not homogenous and there is likely to be variability in substance use between, for instance, different Asian ethnicities, religions, age, gender and locality, future research should ensure, using appropriate sampling strategies and methods, that cultural differences are no longer concealed. 


\section{References:}

Aldridge, J., Measham, F. and Williams, L. (2011) Illegal Leisure Revisited: Changing Patterns of Alcohol and Drug Use in Adolescents and Young Adults. London: Routledge.

Best, D., Manning, V., Gossop, M., Gross, S., and Strang, J. (2006) 'Excessive drinking and other problem behaviours among 14-16 year old schoolchildren'. Addictive Behaviors, 31(8): 1424-1435.

Best, D., Rawaf, S., Rowley, J., Floyd, K., Manning, V. and Strang, J. (2001) 'Ethnic and Gender Differences in Drinking and Smoking among London Adolescents', Ethnicity \& Health, 6:1: 51-57.

Bradby, H. (2007) 'Watch out for the Aunties! Young British Asians' accounts of identity and substance use'. Sociology of Health \& Illness, 29(5): 656-672.

Casadio, P., Fernandes, C., Murray, R. and Di Forti, M. (2011) 'Cannabis use in young people: The risk of schizophrenia'. Neuroscience and Biobehavioral Reviews, 35: 1779-1787.

Coomber, R., Moyle, L. and South, N. (2015) The normalisation of social supply: The social supply of drugs as the "other side" of the history of normalisation. Drugs: Education, Prevention and Policy, Early Online 1-9.

Duff, C. (2005) Party drugs and party people: examining the 'normalization' of recreational drug use in Melbourne, Australia. International Journal of Drug Policy, 16, 3, 161-70.

Fountain, J., Bashford, J. and Winters, M. (2003) Black and minority ethnic communities in England: a review of the literature on drug use and related service provision. London: National Treatment Agency for Substance Misuse and the Centre for Ethnicity and Health.

HSCIC (2015) Smoking, drinking and drug use among young people in England in 2014. London: HSCIC.

Hammersley, R., Marsland, L., Reid, M., and Britain, G. (2003) Substance use by young offenders: the impact of the normalisation of drug use in the early years of the 21st century. London: Home Office (2003)

Hay, G., dos Santos, A. \& Millar, T., (2013) National and Regional Estimates of the Prevalence of Opiate and/or Crack Cocaine Use 2010-11: A summary of key findings. Liverpool: Centre for Public Health, Liverpool John Moore's University.

Home Office (2014) Drug Misuse Declared: Findings from the 2013/14 Crime Survey for England and Wales. London: Home Office.

Jawad, M., McIver, C. and Iqbal, Z. (2014) 'Prevalence and correlates of lifetime waterpipe, cigarette, alcohol and drug use among secondary school students in Stoke-on-Trent, UK: a post hoc cross-sectional analysis', Journal of Public Health, 36(4): 615-621. 
Jayakody, A. A., Viner, R. M., Haines, M. M., Bhui, K. S., Head, J. A., Taylor, S. J. C., and Stansfeld, S. A. (2006) 'Illicit and traditional drug use among ethnic minority adolescents in East London'. Public Health, 120(4): 329-338.

Jivraj, S. (2012) How has ethnic diversity grown 1991-2001-2011? Manchester: Centre on Dynamics of Ethnicity.

Jivraj, S. and Khan, O. (2013) Ethnicity and deprivation in England: How likely are ethnic minorities to live in deprived neighbourhoods? Manchester: Centre on Dynamics of Ethnicity.

Lader, D. (2015) Drug Misuse: Findings from the 2014/15 Crime Survey for England and Wales (Second Edition). London: Home Office.

Measham, F. and Shiner, M. (2009) 'The legacy of 'normalisation': the role of classical and contemporary criminological theory in understanding young people's drug use', International Journal of Drug Policy, 20: 502-8.

Measham, F., Aldridge, J. and Parker, H. (2001) Dancing on Drugs: Risk, health and hedonism in the British club scene. London: Free Association Books.

Moore, K., Dargan, P. I., Wood, D. M. and Measham, F. (2011) 'Do Novel Psychoactive Substances Displace Established Club Drugs, Supplement Them or Act as Drugs of Initiation? The relationship between Mephedrone, Ecstasy and Cocaine', European Addiction Research, 19: 276-82.

Mauseth, K. B., Skalisky, J., Clark, N. E., \& Kaffer, R. (2015). Substance Use in Muslim Culture: Social and Generational Changes in Acceptance and Practice in Jordan. Journal of religion and health, 1-14.

NDTMS (2015) Young people's statistics from the National Drug Treatment Monitoring System (NDTMS) 1 April 2014 to 31 March 2015. London. Public Health England.

ONS (2013) Detailed Characteristics for England and Wales, March 2011. London: ONS.

Parker, H., Aldridge, J. and Measham, F. (1998) Illegal Leisure: The Normalization of Adolescent Recreational Drug Use. London: Routledge.

Parker, H., Williams, L. and Aldridge, J. (2002) 'The Normalization of 'Sensible' Recreational Drug Use: Further Evidence from the North West England Longitudinal Study'. Sociology, 36(4): 941-964.

Patel, K. and Wibberley, C. (2002) 'Young Asians and Drug Use'. Journal of Child Health Care, 6(1): 51-59.

Pearson, G. and Patel, K. (1998) Drugs, Deprivation, and Ethnicity: Outreach among Asian Drug Users in a Northern English City Journal of Drug Issues 28(1): 199-224.

Pearson, G. (2001) 'Normal Drug Use: Ethnographic fieldwork among an adult network of recreational drug users in inner London'. Substance Use and Misuse, 36 (1\&2): 167-200. 
Rodham, K., Hawton, K., Evans, E., and Weatherall, R. (2005) 'Ethnic and gender differences in drinking, smoking and drug taking among adolescents in England: a self-report school-based survey of 15 and 16 year olds'. Journal of Adolescence, 28(1): 63-73.

Rodner-Sznitman, S. (2008) Drug Normalization and the Case of Sweden, Contemporary Drug Problems, June-September, 35, 2-3, 447-80.

Rubino, T. and Parolaro, D. (2008) 'Long lasting consequences of cannabis exposure in adolescence'. Molecular and Cellular Endocrinology, 286S: S108-S113.

Safian, Y. (2013). An analysis on Islamic rules on drugs. International Journal of Education and Research, 1, 1-16.

Seddon, T., Williams, L. and Ralphs, R. (2012) Tough Choices: Risk, Security and Criminalization of Drug Policy. Oxford: Oxford University Press.

Shapiro, G. and Buckley-Hunter, L. (2010) 'What every adolescent needs to know: Cannabis can cause psychosis'. Journal of Psychosomatic Research, 69: 533-539.

Shildrick. T. (2002) 'Young People, Illicit Drug Use and the Question of Normalization', Journal of Youth Studies, 5 (1): 35-48.

Shiner, M. and Newburn, T. (1997) 'Definitely, maybe not? The Normalisation of Recreational Drug use amongst Young People'. Sociology, 31(3): 511-29

Shiner, M. and Newburn, T. (1999) 'Taking tea with Noel: the place and meaning of drug use in everyday life' in N. South (ed.) Drugs, Controls and Everyday Life. London: Sage.

The Muslim Council of Britain (2015) British Muslims in Numbers: A Demographic, Socioeconomic and Health profile of Muslims in Britain drawing on the 2011 Census. London: Muslim Council of Britain.

UK Drug Policy Commission (2010) Drugs and Diversity: Ethnic Minority Groups. London: UK Drug Policy Commission.

Wanigaratne, S., Dar, K., Abdulrahim, D. and Strang, J. (2003) 'Ethnicity and Drug Use: exploring the nature of particular relationships among diverse populations in the United Kingdom', Drugs: Education, prevention and policy, 10(1): 1-17.

Williams, L. (2016) 'Muddy waters? Reassessing the dimensions of the normalisation thesis in $21^{\text {st }}$ century Britain?', Special Issue: The Normalisation Thesis 20 Years On, Drugs: Education, Prevention and Policy.

Williams, L. (2013) Changing Lives, Changing Drug Journeys: Drug taking decisions from adolescence to adulthood. Abingdon: Routledge. 


\footnotetext{
' Drug users accessing drug treatment largely consist of heroin and crack cocaine users. Yet, this population comprises a small proportion of the drug using population. Hays et al. (2013) estimated, for the year 2010-11, there were 298,752 opiate and crack cocaine users in England and Wales. This compares to 2.8 million adults aged 16-59 who reported taking an illicit drug in the past year in 2014/15 (Lader, 2015).

ii See Safian (2013) for a detailed historical analysis of traditional Muslim societies, and attitudes towards and evidence of the use of 'hashish'.

iii Paan is a chewing mixture of areca nut and tobacco that is popular among South Asian populations including Pakistani and Bangladeshi.

iv One of the conclusions arising from the thesis was that gender, class and ethnicity were no longer strong determinants of recreational drug taking (Parker et al., 1998). Since then, it has been argued they remain important for explaining contemporary drugs consumption (see Aldridge et al., 2011; Measham and Shiner, 2009; Measham et al., 2011; Williams, 2013)

v Shildrick's paper crossed in publication with Parker et al. (2002). The latter article re-emphasised how normalisation is a qualified concept explaining specific styles of drugs consumption and attitudes towards them. It also highlighted the diversity of experience and views among the Illegal Leisure sample, including that there were some steadfast abstainers who did not accept recreational drug use.

vi Although the Illegal Leisure sample was initially representative of the areas in which data were collected, and included respondents from different ethnic backgrounds, over time attrition resulted in it becoming less representative of Asian British participants (Aldridge et al., 2011; Parker et al., 1998, 2002), and, therefore, the concept has not been sufficiently applied to this population.

vii Pseudonyms have been used for the area and drug service where we collected our data.

viii Observations of the outreach project, including shadowing the outreach team in the community, in educational establishments, and during one-to-one meetings with clients, were also carried out, however, analysis of these data is not presented.

${ }^{\text {ix }}$ Drugs refers to both traditional illegal substances, such as cannabis, ecstasy and cocaine, and novel psychoactive substances aka legal highs, for instance, synthetic cannabinoids.

${ }^{x}$ Skunk is a more potent strain of herbal cannabis.

${ }^{x i}$ Not forbidden or proscribed by Islamic law.
} 